Les médecins veulent pouvoir soigner leurs patients de la meilleure manière possible et s'engagent jour après jour pour garantir la qualité des soins. Cette tâche essentielle qui est depuis toujours une évidence pour le corps médical bénéficie d'une base institutionnelle depuis novembre 2012, grâce à l'Académie suisse pour la qualité en médecine (ASOM) fondée par la FMH. A I'automne 2016, I'ASOM s'est dotée d'une charte qualité qui souligne l'engagement des organisations médicales notamment en matière de transparence. Cet engagement, facultatif, repose sur une démarche qui prend corps sur le terrain («bottom-up»). Mais la clé du succès réside autant dans le développement des structures existantes que dans le soutien de nouvelles avancées grâce à des conditions-cadres appropriées. Pour y parvenir, une collaboration constructive entre les prestataires et les assureurs constitue un facteur de réussite essentiel.

\title{
Comment la qualité a investi la prise en charge médicale?
}

\section{Bernhard Güntert}

Prof. Dr oec. / MHA, membre de la direction de I'association d'assureurs curafutura et responsable du domaine Qualité

Les efforts en vue de garantir la qualité des soins sont vieux comme le monde. La protection des patients et l'engagement des professionnels de santé à appliquer des méthodes thérapeutiques efficaces ont toujours été au cœur des préoccupations. Dès le XVIII siècle avant J.C., Hammurabi prévoit, dans son Codex, des sanctions draconiennes pour les médecins qui blessent leurs patients ou utilisent des ustensiles sales [1]. On ignore toutefois si, ou comment ces sanctions ont été efficaces. Il n'en reste pas moins que des problèmes de qualité subsistent aujourd'hui. Dans sa publication To Err is Human [2], l'Institute of Medicine révèle qu'un nombre surprenant de patients souffrent des suites d'un traitement. Des études complémentaires menées dans différents pays ont malheureusement confirmé ce résultat (entre autres [3]). En Suisse aussi, on estime - selon les hypothèses choisies - entre 700 et 3000 cas de décès évitables après traitements hospitaliers [4].

\section{La médecine en tant que science et profession}

La médecine est une science et une profession. On parle de profession lorsque le domaine d'application et les prestations fournies, la formation initiale et postgraduée, et l'admission à la formation professionnelle sont définies par la corporation elle-même qui, par ailleurs, mène des recherches sur ses propres activités [5]. Ces caractéristiques s'appliquent à la médecine mais la législation actuelle en matière de santé et de formation relativise cette autodétermination.

L'autorégulation de la profession inclut - et incluait également la définition de la qualité. Le modèle d'analyse de la qualité développé par le Dr Avedis Donabedian dans les années 60 a constitué une base importante dans ce domaine. Dans une grille à deux dimensions, il a distingué d'une part la qualité des structures, des processus et des résultats et, d'autre part - ce qui est moins connu, mais qui joue un rôle de plus en plus important - la qualité technique, interpersonnelle et morale ou éthique [6]. En raison de sa plausibilité, ce modèle continue encore aujourd'hui de dominer le débat sur la qualité des soins, même si le rapport de causalité (postulat) entre structures, processus et résultats n'a pas pu être démontré de manière empirique, sauf dans les cas de différences structurelles très importantes. Une dimension supplémentaire s'ouvre avec la définition suivante de l'Institute of Medicine: «Quality of care is the degree to which health services for individuals and populations increase the likelihood of desired health outcomes and are consistent with current professional knowledge» [7]. Cette définition pose un lien, d'une part, avec la preuve scientifique et donc le rôle de plus en plus important de la qualité du diagnostic et de l'indication et, d'autre part, avec la population. Cette référence à la population relativise le pouvoir de définition de la profession médicale en ce qui concerne la qualité des prestations car les aspects chers à la population ou aux patients mais aussi aux politiques et aux assureurs s'invitent au débat. De plus, en finançant le système de santé, la politique et les assureurs exercent une influence décisive sur les ressources disponibles et l'accès aux soins. En Suisse, la loi sur l'assurancemaladie (LAMal) promulguée en 1996 a également limité l'autorégulation de la profession. L'art. 58 LAMal a conféré au Conseil fédéral la possibilité de contrôler la garantie de la qualité et d'y participer activement. Ce dernier s'est toutefois contenté à l'époque de charger les partenaires tarifaires de régler entre eux la question de la qualité par voie contractuelle. Or, pour diverses raisons, ces partenaires n'ont pas véritablement tiré parti du potentiel d'une telle délégation de compé- 
tence. L'une des conséquences est que l'annexe $6 \mathrm{du}$ TARMED qui était censée régler les exigences spécifiques en matière de qualité n’a jamais été élaborée. D'autres aspects de l'autorégulation des professionnels de santé ont également été relativisés. Ainsi, l'Etat règle aujourd'hui pratiquement à lui seul la formation et l'admission des professionnels de santé (art. 35 à 40 LAMal).

\section{Garantie et développement de la qualité}

Dix ans après l'entrée en vigueur de la LAMal, le Parlement a demandé de contrôler la mise en œuvre de l'assurance-qualité dans le domaine de la santé [8]. Les efforts des partenaires tarifaires ont été jugés insuffisants. Par la suite, la Confédération a élaboré une Stratégie "Qualité» [9] dans laquelle elle a défini le leadership dans ce domaine. Elle voulait créer les conditions nécessaires à la garantie et au développement de la qualité, définir des programmes d'action et en assurer la mise en œuvre. En 2014, elle a proposé de regrouper ces activités et celles concernant l'évaluation des technologies dans un «Centre pour la qualité dans l'assurance obligatoire des soins» [10]. Cette démarche a été rejetée par la majorité des acteurs de la santé. En conséquence, la Confédération a confié la responsabilité des HTA à l'OFSP et joue ainsi un rôle actif dans la garantie de la qualité du catalogue des prestations [11]. Fin 2015, le Conseil fédéral a soumis au Parlement un nouveau projet de révision des articles 58 et 59 de la LAMal se concentrant principalement sur la création d'une Commission fédérale pour la qualité dans l'assurance-maladie et la création d'une unité d'organisation de l'OFSP chargée de la mise en œuvre des activités. Le financement de la Stratégie "Qualité» de la Confédération devait être assuré par une augmentation des primes de l'assurance obligatoire des soins. La commission d'examen préalable du Conseil des Etats a récemment décidé de ne pas entrer en matière sur le projet. Et son homologue du Conseil national a demandé l'élaboration de plusieurs variantes (commission extraparlementaire, organisme ad hoc indépendant). Les débats sont en cours.

L'actuelle discussion sur la révision des articles 58 et 59 LAMal, intitulée "Renforcement de la qualité et de l'économicité», suscite la controverse et fait apparaître les différences de points de vue entre les professionnels de santé, la Confédération, les cantons et les assureurs dans ce dossier. Fondamentalement, il s'agit de répondre aux questions suivantes: la qualité des prestations peutelle être imposée par le haut, à savoir par des mesures législatives, à l'instar des branches où la qualité est principalement technique? Ou bien, les prestations de santé ont-elles un caractère particulier en raison de leurs aspects interpersonnels et éthiques majeurs? La qualité ne devrait-elle pas être développée par le bas (sur le terrain) en concertation professionnelle (ou aujourd'hui plus exactement interprofessionnelle) avec la participation des patients et des assureurs? L'Etat ne devrait-il pas concentrer ses efforts pour garantir la qualité des prestations en mettant en place des structures ou des incitatifs susceptibles de faciliter, d'encourager ou de rendre nécessaire le débat sur la qualité entre les partenaires tarifaires et les différents acteurs de la santé?

\section{Le rôle des professionnels de santé}

Les expériences observées en Suisse et à l'étranger montrent que les professionnels de santé sont généralement le moteur du développement de la qualité dans le domaine de la santé mais que ce développement peut être amélioré par des conditions-cadres adéquates responsabilisant les acteurs. L'introduction et la généralisation du système DRG (forfaits par cas liés au diagnostic) comme modèle de financement des hôpitaux dans différents pays ont contribué à une plus grande prise de conscience des processus et des standards de traitement de la part des professionnels de santé, permettant ensuite le développement et l'évaluation d'indicateurs mesurables de la qualité des processus et des résultats [12]. Suite aux expériences acquises aux Etats-Unis et dans quelques autres pays, les hôpitaux allemands ont été tenus de mettre en place la gestion de la qualité dès 1998, avant même l'introduction du système G-DRG [13], mais les hôpitaux ont été libres de choisir leur modèle (KTQ, proCumCert, IJCQ, EFQM, ISO, etc.). Depuis 2005, les hôpitaux allemands ont l'obligation de publier régulièrement sur Internet leurs indicateurs qualité sous forme de rapports de qualité. Les normes prescrites par la Commission fédérale commune (Gemeinsamer Bundesausschuss) pour ces rapports de qualité sont continuellement développées. Elles ont permis d'encourager la transparence dans le domaine des soins hospitaliers et de multiplier les moteurs de recherche pour hôpitaux. En Suisse aussi, la Confédération a défini sa Stratégie «Qualité» avant l'introduction du système DRG (2012) [9]. Ensuite, deux projets pilotes nationaux ont été lancés dans une première phase: «La sécurité en chirurgie» et «La sécurité de la médication aux interfaces». La Fondation pour la sécurité des patients (www.securitedespa tients.ch), fondée dès 2003 , bénéficiant d'un large soutien, a été chargée de leur mise en œuvre. Une autre initiative dans le domaine des soins hospitaliers a été la création de l'Association ANQ (www.anq.ch) par les cantons, $\mathrm{H}+$ et les assureurs ainsi que la conclusion du 
contrat qualité national qui a permis de mesurer et de publier divers indicateurs de qualité du secteur hospitalier. La Suisse a ainsi jeté les premiers jalons de la compétitivité entre les hôpitaux via la qualité même si pour l'instant, un arrêt du tribunal (ATAF 2014/36) empêche que la qualité puisse influer sur les prix de base (baserate) du système DRG [14].

\section{Le secteur ambulatoire}

Dans la plupart des pays, les efforts en vue du développement et de la garantie de la qualité ont été moins intensifs dans le secteur ambulatoire. En Suisse, suite aux vives critiques qui leur ont été adressées, les organisations de soins intégrés (Managed Care) apparues après la révision de la LAMal de 1996 se sont penchées activement sur la qualité et les modèles de gestion de la qualité pour les cabinets et les réseaux de médecins. La Fondation EQUAM (www.equam.ch) pour la démarche qualité dans les cabinets médicaux a été créée en 1999 et se concentre principalement sur le Managed Care. Elle développe des programmes de qualité et des modules axés sur les maladies qu'elle adapte continuellement aux nouvelles exigences (p. ex. soins intégrés) et à l'évaluation européenne des cabinets médicaux (EPA) (www.epa-qm.de). Mise en place initialement par des médecins, l'EQUAM réunit aujourd'hui des organisations de patients et des assureurs. Elle est largement reconnue (Confédération, ASSM, etc.), respecte les critères de certification de l'Académie suisse des sciences médicales et elle est l'un des organismes de certification accrédités par la Confédération [15]. Hormis l'EQUAM, le corps médical a créé plusieurs autres programmes de gestion de la qualité pour les soins ambulatoires avec des objectifs différents, comme p. ex. QBM (www.politikpatient.ch), Good Medical Practice (GMP) (http://www.sqs.ch/fr/Offre-de-Prestation/Produits/ Labels/Good-Medical-Practice ${ }^{\circledR} /$ L.GMP/), MehrFachArzt MFA (www.mehrfacharzt.ch). Tous ces modèles et labels contribuent à une prise de conscience de la qualité, veillent au respect des objectifs qualité et en mesurent certains indicateurs. Leurs principaux objectifs consistent en règle générale à sensibiliser les médecins et les collaborateurs, à clarifier les structures de base des cabinets médicaux et à mettre en place un benchmark afin d'initier des processus d'amélioration de la qualité dans les cabinets. Comme leur objectif premier ne consiste pas à créer plus de transparence en matière de qualité, ces initiatives ne peuvent pas, sous leur forme actuelle, servir de base pour susciter la compétitivité via la qualité dans le domaine ambulatoire. Les différents concepts étant difficilement comparables entre eux, l'Initiative Q fondé en 2013 par plusieurs de ces labels devait offrir une plateforme visant un regroupement et une certaine harmonisation. Mais cette initiative est restée sans suite. La question de l'harmonisation a été reprise par l'ASQM et mise en œuvre en 2016 avec la Charte qualité (http://www.fmh.ch/fr/ asqm/charte_qualite.html) signée entre-temps par 67 organisations de médecins soulignant leur engagement en matière de transparence, de durabilité et de responsabilité.

Même si la Fondation pour la sécurité des patients se focalise davantage sur les soins hospitaliers, elle a identifié ces dernières années différents problèmes de qualité dans le secteur ambulatoire, notamment en ce qui concerne la sécurité de la médication, la gestion des risques, les problèmes aux interfaces et la gestion des erreurs. Elle a donc élaboré des solutions possibles et des concepts de mise en œuvre. Il semble que la transparence en matière de qualité suscite l'arrivée sur le marché d'un nombre toujours plus important de prestataires proposant p. ex. des plateformes d'évaluation des médecins ou des cabinets médicaux (cf. entre autres: http://docapp.ch) ou créant une nouvelle offre d'informations qualité par une utilisation innovante des technologies ICT. Le projet «Patient Centered Outcome Registry" (PCOR) de l'ASQM/FMH mené avec la Poste (www.fmh.ch/fr/asqmASQM) en est une bonne illustration. Ce projet se distingue de la plupart des autres concepts de gestion de la qualité car il met au centre la question de savoir dans quelle mesure les attentes du traitement ont été remplies conformément aux objectifs définis préalablement entre le patient et les professionnels de santé et qu'il les met ensuite en lien avec les données cliniques.

Pour les tiers (patients, assureurs), les labels susmentionnés ont perdu un peu de leur importance ces dernières années. Il est en effet de plus en plus fréquent que les assureurs-maladie définissent leurs propres exigences en matière de qualité au niveau des structures et des processus, et progressivement aussi des résultats, par des conventions de soins intégrés (Managed Care) et déterminent la documentation pour justifier de la qualité. De ce fait, les partenaires tarifaires ne cessent finalement de remplir un peu plus leur mandat légal.

\section{Promouvoir le développement et la transparence de la qualité}

La révision actuelle de l'art. 58 LAMal est censée promouvoir le développement et la transparence de la qualité. La qualité des prestations ne relève plus que des seuls professionnels de santé. Lors du choix du médecin, les patients se basent de moins en moins sur le bouche-à-oreille ou le hasard, mais souhaitent - 
comme c'est le cas dans de nombreux autres domaines - prendre leurs décisions en s'appuyant sur des informations objectives. Les produits d'assurancemaladie englobent, eux aussi, de plus en plus souvent une prestation de conseil. Les assureurs-maladie ont donc également besoin d'informations qualité objectives. Tandis que les hôpitaux disposent de plus en plus de ce type d'informations, elles font encore largement défaut dans le secteur ambulatoire. Ces informations seront pourtant de plus en plus exigées, à la fois par le «nouveau patient» $[16,17]$ et les assureurs. Les professionnels de santé sont donc appelés, d'une part, à faire la transparence sur les modèles existants et à participer au développement d'indicateurs qualité adéquats qui serviront au développement continu de leur propre qualité et, d'autre part, à aider les utilisateurs dans l'interprétation [18]. Dans le cas contraire, il est probable que les indicateurs, leur mesure et leur publication soient imposés par le haut («top down»).

Le projet de révision de la loi montre clairement la volonté de la Confédération de changer la situation actuelle en favorisant la compétitivité en matière de qualité des soins. Plusieurs points doivent cependant être respectés: le risque existe qu'avec des propositions de solutions centralisatrices, des activités et mesures qualité imposées et l'obligation de contrats qualité nationaux, on freine davantage qu'on encourage les initiatives qualité existantes. Il est ainsi apparu en Allemagne que les activités et les mesures qualité exigées par la Commission fédérale commune et mises en place par les associations de médecins conventionnés n'avaient pas engendré d'amélioration tangible [19]. D'un côté, de nombreux professionnels de santé doutent de l'utilité de telles mesures; de l'autre, la haute complexité, les nombreuses variables et l'hétérogénéité des interventions qualité rendent la base d'évaluation insuffisante pour des déclarations d'ordre général. Pour ces raisons, les activités qualité régionales ou liées à des groupes de prestations ou de patients continuent de jouer un rôle important dans le développement de la qualité des soins. La "Stratégie qualité des soins hospitaliers dans le canton de Zurich 2017-2022» (http://www.vzk.ch/ fileadmin/PDF/Allgemein/QualitaetsManagement/ Qualitätsstrategie_Kanton_Zürich.pdf) en est une parfaite illustration. De telles initiatives font avancer le débat sur la qualité entre les différents acteurs, débouchent sur de nouveaux indicateurs qualité et d'autres projets pilotes, et peuvent, si elles font leurs preuves, servir de base à des contrats qualité natio- naux. Il est dès lors souhaitable que le législateur laisse explicitement la porte ouverte à d'autres développements que les seuls contrats qualité nationaux dans la révision de l'art. 58 LAMal. Il convient d'encourager les efforts des professionnels de santé en matière de qualité, pas de les entraver. Il faut en effet éviter que les acteurs de la santé se retirent de ces activités faute de contrats qualité (ou à la formulation abstraite). Dans cet objectif, il est impératif que le législateur confie la mise en œuvre des activités qualité à une organisation indépendante de l'administration fédérale, qui impliquera les professionnels de santé et les patients mais aussi les assureurs et les cantons dans la responsabilité structurelle (p. ex. sous forme d'une fondation, d'une association ou d'une SA) et dans la prise de décision stratégique [18].

\section{Références}

1 Harke JD (2007). Das Sanktionssystem des Codex Hammurapi, Würzburger rechtswisseschaftl. Schriften 70, Würzburg.

2 Kahn L, Corrigan J, Donaldson M. To err is human - building a safer health system, National Academic Press (2000), Washington DC.

3 OCDE (2017a). Tackling Wasteful Spending on Health, OECD Publishing, Paris.

4 Trageser J, Vettori A, Iten R, Crivelli L (2012). Effizienz, Nutzung und Finanzierung des Gesundheitswesens, Akademien der Wissenschaften Schweiz, Berne.

5 Freidson E (2013). Professionalism: The Third Logic, John Wiley \& Sons, London.

6 Donabedian A (2003). An Introduction to Quality Assurance in Health Care, Oxford University Press, New York.

7 IOM Insitute of Medicine (1990). Medicare: a strategy for quality assurance, Vol 1, National Academic Press, Washington DC.

8 CPA (2008). Le rôle de la Confédération dans la garantie de la qualité selon la LAMal, rapport du Contrôle parlementaire de l'administration à l'attention de la Commission de gestion du Conseil des Etats, FF 2008, 7797-888.

9 OFSP (2009). Stratégie fédérale en matière de qualité dans le système de santé, DFI, Berne.

10 OFSP (2014). Avant-projet de loi fédérale sur le centre pour la qualité dans l'assurance obligatoire des soins, DFI, Berne.

11 OFSP (2015). Fiche Info «Programme HTA / Stratégie HTA et procédure d'autorisation", DFI, Berne, août 2015.

12 Nylenna M, Bjertnages O, Sperre Saunes I, Lindahl AK (2015). What is Good Quality of Health Care?, in: Professions and Professionalism, Vol 5, No 1, 1-16.

13 Güntert B, Offermanns G (2001). Qualitätsmanagementmodelle für das Gesundheitswesen, dans: lögd (éd.), Qualitätsmanagement im ÖGD, lögd, Tome 9, Bielefeld, p. 13-33.

14 LFPr 2014/36; Décision de principe du Tribunal administratif fédéral du 11 septembre 2014.

15 ASSM (2011). Certification dans le contexte médical, Recommandations de l'Académie suisse des sciences médicales, Bâle.

16 Oertle D (2016). Les médecins de famille sont en voie de disparition. Et alors? Bull Méd Suisses, 2016;97(51-52):1804-5.

17 Wolff E (2017). De la critique des patients. Bull Méd Suisses 2017;98(13):422.

18 OCDE (2017b). Caring for Quality in Health - lessons learnt from 15 reviews of health care quality, OECD Publishing, Paris.

19 Sorgenfrei JH (2015). Qualitätsmanagement in der vertragsärztlichen Versorgung - Evaluation durch den Gemeinsamen Bundesausschuss, Diplomica Verlag, Hambourg. 